\title{
INFLUENCE OF A ONE-ION ANISOTROPY ON STABILIZATION OF THE LONG-RANGE MAGNETIC ORDER IN TWO-DIMENSIONAL FERROMAGNET
}

\author{
YU.N. Mitsay ${ }^{a}$, YU.A. Fridman ${ }^{a}$, D.V. SPIRIN ${ }^{a}$ AND M.S. Kochmański ${ }^{b}$ \\ ${ }^{a}$ V.I. Vernadsky Tavricheskii National University \\ 333007, Yaltinskaya st., 4, Simferopol, Ukraine \\ ${ }^{b}$ Institute of Physics, University of Rzeszów, Rejtana 16A, 35-310 Rzeszów, Poland
}

(Received October 19, 1999)

\begin{abstract}
In the paper the possibility of stabilization of the long-range magnetic order in a two-dimensional ferromagnetic with one-ion easy-plane anisotropy is explored. It is shown that at the presence of a small anisotropy in a two-dimensional ferromagnetic the long-range magnetic order is stabilized by magnetoelastic interaction. If one-ion anisotropy is comparable or even exceeds the exchange interaction in the system there exists a long-range non-vectorial quadrupolar tensorial magnetic order.
\end{abstract}

PACS numbers: $75.10 .-\mathrm{b}, 75.30 . \mathrm{Kz}$

\section{Introduction}

The behaviour of two-dimensional isotropic ferromagnets or ferromagnetics with one-ion easy-plane type anisotropy differs from behaviour of three-dimensional magnetics. This difference lies in the lack of the long-range magnetic order, and also in the existence of the phase transition of a special type: at temperatures lower than the critical temperature in the system there takes place coupling of solitons resulting in arising vortex-antivortex pairs (the Berezinsky phase), and with the increase in temperature the vortex pairs dissociate [1-4].

As was shown in $[5,6]$, the account of relativistic interactions like magnetodipole or magnetoelastic interaction leads to modification of the law of a dispersion of elementary excitations. With the account of magnetodipole interaction the law of dispersion turns to be a square root one and with the account of magnetoelastic interaction in a spectrum there is a magnetoelastic gap. Such reorganization of spectra essentially reduces thermal fluctuations in the system and by that stabilizes the long-range magnetic order. 
Such approach to examination of the behaviour of two-dimensional ferromagnetics is valid when the quantum fluctuations of spin are small and the basic role is played by thermal fluctuations, i.e. in the case when one-ion anisotropy is small $\left(\beta \ll J_{0}\right.$, where $\beta$ is a constant of anisotropy, $J_{0}$ is a zero Fourier-component of exchange integral).

Another situation can be realized in two-dimensional magnetics with great value of one-ion anisotropy $\left(\beta / 4 \geq J_{0}\right)$, the so-called strongly anisotropic magnetics. In such systems interesting quantum effects are predicted, such as the existence of non-magnetized (quadrupolar) phases with zero mean value of spin (but with non-zero mean value of bilinear combinations of projections of spin), extra branches of magnons, etc. [7].

Let us explore the properties of a two-dimensional easy-plane ferromagnetic, assuming that the magnetoelastic interaction stabilizes the long-range magnetic order. We shall consider two different cases: the case of small and great one-ion anisotropy. The account of great one-ion anisotropy leads to appearance of various pure quantum effects, that in its turn requires the use of an adequate mathematical means. It is convenient in this situation to use the technique of Hubbard's operators $[7,8]$.

\section{The model and basis results}

We shall present the Hamiltonian of the system in study in the following form:

$$
\begin{aligned}
H= & -\frac{1}{2} \sum_{n, n^{\prime}} J\left(n-n^{\prime}\right) S_{n} S_{n^{\prime}}+\frac{\beta}{2} \sum_{n}\left(S_{n}^{y}\right)^{2} \\
& +\lambda \sum_{n}\left[\left(S_{n}^{x}\right)^{2} u_{x x}+\left(S_{n}^{z}\right)^{2} u_{z z}+\left(S_{n}^{x} S_{n}^{z}+S_{n}^{z} S_{n}^{x}\right) u_{x z}\right] \\
& +\int \mathrm{d} v \frac{E}{2\left(1-\sigma^{2}\right)}\left[u_{x x}^{2}+u_{z z}^{2}+2 \sigma u_{x x} u_{z z}+2(1-\sigma) u_{x z}^{2}\right],
\end{aligned}
$$

where $J\left(n-n^{\prime}\right)>0$ is an exchange integral, $S_{n}^{i}$ is a spin operator in site $n, \beta>0$ is a constant of one-ion anisotropy, $\lambda$ is a constant of magnetoelastic interaction, $u_{i j}$ is a symmetric part of components of deformations tensor, $E$ is a modulus of elasticity, $\sigma$ is a Poisson coefficient.

The system described by Hamiltonian (1) represents an easy-plane ferromagnetic ( $X O Z$ is a basis plane). The two-dimensionality of a system, as well as earlier, is taken into account in elastic and magnetoelastic energies $\left(u_{z z}=u_{z y}=u_{z x}=0\right)$. To simplify further calculations we shall assume that the spin of a magnetic ion $S=1$.

Separating the mean field in the exchange part of (1) we shall receive the one-ion Hamiltonian

$$
\begin{aligned}
& H_{0}(n)=-J_{z} S_{n}^{z}+\frac{\beta}{2}\left(S_{n}^{y}\right)^{2}+\lambda u_{x x}\left(S_{n}^{x}\right)^{2}+\lambda u_{z z}\left(S_{n}^{z}\right)^{2} \\
& \quad+\lambda u_{x z}\left(S_{n}^{x} S_{n}^{z}+S_{n}^{z} S_{n}^{x}\right)
\end{aligned}
$$

where $J_{z}=J_{0}\left\langle S^{z}\right\rangle$. The solution of one-ion problem with Hamiltonian (2) enables us to obtain the energy levels of a magnetic ion 


$$
\begin{aligned}
& E_{1}=\frac{\lambda}{2}\left(u_{x x}^{(0)}+2 u_{z z}^{(0)}\right)+\frac{\beta}{4}-\chi, \quad E_{0}=\lambda u_{x x}^{(0)}+\frac{\beta}{2}, \\
& E_{-1}=\frac{\lambda}{2}\left(u_{x x}^{(0)}+2 u_{z z}^{(0)}\right)+\frac{\beta}{4}+\chi, \quad \chi^{2}=J_{z}^{2}+\left(\frac{\beta}{4}+\frac{\lambda}{2} u_{x x}^{(0)}\right)^{2} .
\end{aligned}
$$

The spontaneous deformations $u_{i j}^{(0)}$ are determined from the requirement of a minimum of a free-energy density, and have the form

$$
u_{x x}^{(0)}=-\frac{\lambda}{E} \frac{1-2 \sigma}{2}, \quad u_{z z}^{(0)}=-\frac{\lambda}{E} \frac{2-\sigma}{2}, \quad u_{x z}^{(0)}=0 .
$$

The connection of spin operators with Hubbard's ones [9] looks like

$$
\begin{aligned}
& S_{n}^{+}=\sqrt{2} \cos \delta \cdot\left(X_{n}^{10}+X_{n}^{0-1}\right)+\sqrt{2} \sin \delta \cdot\left(X_{n}^{01}-X_{n}^{-10}\right), \quad S_{n}^{-}=\left(S_{n}^{+}\right)^{+}, \\
& S_{n}^{z}=\cos 2 \delta \cdot\left(H_{n}^{1}-H_{n}^{-1}\right)-\sin 2 \delta \cdot\left(X_{n}^{1-1}+X_{n}^{-11}\right), \\
& \cos \delta=\left(\frac{\lambda u_{x x}^{(0)}}{2}-\frac{\beta}{4}\right) / \sqrt{\left(\chi-J_{z}\right)^{2}+\left(\lambda u_{x x}^{(0)} / 2-\beta / 4\right)^{2}}
\end{aligned}
$$

where $X^{M^{\prime} M}=\left|\psi\left(M^{\prime}\right)\right\rangle\langle\psi(M)|$ are Hubbard's operators, describing the transition of a magnetic ion from a state $M^{\prime}$ to a state $M$.

From (4) it is easy to find a magnetization per unit $\left(\left\langle S^{z}\right\rangle\right)$, which at low temperatures is equal to

$\left\langle S^{z}\right\rangle \cong \cos 2 \delta$.

This equation on $\left\langle S^{z}\right\rangle$ has the following solutions:

$$
\begin{aligned}
& \left\langle S^{z}\right\rangle=0, \\
& \left\langle S^{z}\right\rangle=\sqrt{1-\left(\frac{\beta}{4 J_{0}}\right)^{2}} .
\end{aligned}
$$

For the case of small anisotropy the solution (6) is valid. If $\beta / 4 \geq J_{0}$, the second solution is non-physical, and it is necessary to consider solution (5). Thus, in strongly anisotropic easy-plane magnetics even at low temperatures the magnetization is equal to zero. However in this case the quadrupolar order parameter $q=3\left\langle\left(S^{z}\right)^{2}\right\rangle-S(S+1)$ differs from zero and at finite temperatures is equal to 1 . This parameter weakly depends on temperature, and tends to zero only at $T \rightarrow \infty$ :

$$
\begin{aligned}
\left\langle\left(S^{z}\right)^{2}\right\rangle & =\left\langle H^{1}+H^{-1}\right\rangle=\frac{\exp \left(-E_{1} / T\right)+\exp \left(-E_{-1} / T\right)}{\exp \left(-E_{1} / T\right)+\exp \left(-E_{0} / T\right)+\exp \left(-E_{-1} / T\right)} \underset{T \rightarrow \infty}{\longrightarrow} \frac{2}{3}, \\
q & =3\left\langle\left(S^{z}\right)^{2}\right\rangle-S(S+1) \underset{T \rightarrow \infty}{\longrightarrow} 0
\end{aligned}
$$

Thus, it is possible to assume that in the considered system the quadrupolar phase (QU) is realizable and remains stable at wide temperature interval [8]. 
To prove the validity of the expressed above statement, let us explore the behaviour of fluctuation of magnetization. To evaluate its value it is necessary beforehand to calculate the spectra of elementary excitations of the system.

Since in our model the magnetoelastic interaction is taken into account, in this case it is impossible to consider magnetic (magnons) and elastic excitations (phonons) as independent one, because in the system there will be realized hybridised excitation-magnetoelastic waves [10].

The spectra of elementary excitations of a system are determined by the poles of Green's function, which we shall define as follows:

$$
G^{\alpha \alpha^{\prime}}\left(n, \tau ; n^{\prime}, \tau^{\prime}\right)=-\left\langle\hat{T} X_{n}^{\alpha}(\tau) X_{n^{\prime}}^{\alpha^{\prime}}\left(\tau^{\prime}\right)\right\rangle \text {. }
$$

Here $\hat{T}$ is a time-ordering operator, $X_{n}^{\alpha}(\tau)$ is a Hubbard operator in Heisenberg's representation. We shall carry out further evaluations in the mean field approximation, therefore further we need only cross part of an exchange Hamiltonian, which looks like

$$
H_{\mathrm{int}}^{\perp}=-\frac{1}{2} \sum_{n, n^{\prime} \alpha, \beta} J_{\alpha \beta}^{\perp}\left(n-n^{\prime}\right) X_{n}^{\alpha} X_{n^{\prime}}^{\beta},
$$

where

$$
J_{\alpha \beta}^{\perp}\left(n-n^{\prime}\right)=J\left(n-n^{\prime}\right)\left\{\gamma_{\|}(\alpha) \gamma_{\|}(\beta)+\frac{1}{2}\left[\gamma_{\perp}(\alpha) \gamma_{\perp}^{*}(-\beta)+\gamma_{\perp}^{*}(-\alpha) \gamma_{\perp}(\beta)\right]\right\} .
$$

The functions $\gamma_{\|(\perp)}(\alpha)$ are determined from the connection of spin operators with Hubbard's operators. The dispersion equation of magnetoelastic waves is described in [10]. The quasimagnon spectrum obtained from this equation looks like

$$
\omega^{2}(k)=\left[b_{0}+\frac{\beta}{4}+\chi-J_{k}(1-\sin 2 \delta)\right]\left[b_{0}+\frac{\beta}{4}+\chi-J_{k}(1+\sin 2 \delta)\right],
$$

where $b_{0}=3 \lambda^{2} / 4 E$.

In the case $\beta \ll J_{0},\left\langle S^{z}\right\rangle \approx 1$ (small anisotropy), we obtain the expression

$$
\omega^{2}(k)=\left(b_{0}+\alpha k^{2}\right)\left(b_{0}+\frac{\beta}{2}+\alpha k^{2}\right),
$$

where $\alpha=J_{0} R_{0}^{2}, R_{0}$ is a radius of interaction.

For the case of great one-ion anisotropy $\left(\beta / 4 \geq J_{0},\left\langle S^{z}\right\rangle=0\right)$ we have

$$
\omega^{2}(k)=\left(\frac{\beta}{2}+a_{0}\right)\left(\frac{\beta}{2}+a_{0}-2 J_{0}+2 \alpha k^{2}\right),
$$

where $a_{0}=\lambda^{2}(1+\sigma) / 2 E$ is a parameter of magnetoelastic interaction.

The expressions (9), (10) determine the spectra of quasimagnons at various values of the constant of one-ion anisotropy. A quasiphonon spectrum ( $t$-polarization, $k \| O Z$ ), obtained from the solution of the dispersion equation, describes only weak renormalization of the velocity of sound

$$
\omega_{2}^{2}(k)=\omega_{t}^{2}\left(1-\frac{4 a_{0}}{\beta}\right)
$$

(it is supposed that $\beta \gg \lambda$ ). 
As is evident from expressions (10), (11), the spectra of elementary excitations remain stable in all temperature intervals, i.e. there is no phase transition in the system.

Let us explore the fluctuations of a magnetic moment in the basis plane, for example $\left\langle\left(S^{x}\right)^{2}\right\rangle$. We shall use the bosonization method [11] and rewrite the operator $S^{x}$ in terms of rising and lowering Bose operators $a_{n}^{+}, a_{n}$. For the fluctuation of a magnetic moment we obtain

$$
\left\langle\left(S^{x}\right)^{2}\right\rangle=1-\left\langle a_{n}^{+} a_{n}\right\rangle .
$$

It is possible to present the expression included in (12), in the following form:

$$
\frac{1}{N} \sum_{n}\left\langle a_{n}^{+} a_{n}\right\rangle=\frac{1}{(2 \pi)^{2}} \int_{0}^{\infty} \frac{\left(u_{k}^{2}+v_{k}^{2}\right) k \mathrm{~d} k}{\exp \left[\omega_{1}(k) / T\right]-1},
$$

where $u_{k}$ and $v_{k}$ are the coefficients of a $u-v$-transformation, and $u_{k}^{2}+v_{k}^{2}=$ $\left(E_{10}-J_{0}\right) / \omega(k)$. One can easily see that this integral converges in a lower limit.

At $\beta \ll J_{0}$ the Curie temperature weakly depends on the value of an anisotropy constant, and the decisive role is still played by magnetoelasticity

$$
T_{\mathrm{C}} \approx \frac{4 \pi \alpha}{\ln \frac{4 \pi \alpha}{\sqrt{b_{0}\left(b_{0}+\beta / 2\right)}} .}
$$

At $\beta \gg J_{0}$ and $k \rightarrow 0$ the integral (11) exponentially tends to zero. Thus, the thermal fluctuations in strongly anisotropic easy-plane ferromagnetic at arbitrary temperatures remain finite and small. However, the Curie temperature of such system tends to infinity. Indeed, as in the quadrupolar phase in the plane $X O Z$ there is no chosen quantization axis, then it is possible to accept $\left\langle\left(S^{z}\right)^{2}\right\rangle$ as a value describing fluctuation of a magnetic moment, which, as is obvious from (7), is always greater than zero. If we define the Curie temperature as the temperature, at which the order parameter $q$ is equal to zero, still from (7), it is possible to see that $T_{\mathrm{C}} \rightarrow \infty$.

\section{Conclusions}

The obtained results testify that the behaviour of $2 \mathrm{D}$ strongly anisotropic easy-plane magnetics is specific and significally differs from the behaviour of weakly anisotropic systems.

In weakly anisotropic systems the long-range magnetic order is stabilized by magnetoelastic interaction and the one-ion anisotropy weakly affects the magnitude of fluctuations of a magnetic moment.

In strongly anisotropic magnetics the long-range magnetic order is also realized, however this order is not a ferromagnetic, but quadrupolar one. Thus, the spectra of elementary excitations remain stable in all intervals of temperature, i.e. the system does not undergo the phase transition. At first sight, such behaviour of a system contradicts a number of theorems describing the behaviour of two-dimensional magnetics. According to these theorems in a two-dimensional ferromagnetic there is no long-range magnetic order. However then it was found that in two-dimensional easy-plane magnetics nevertheless there is an original phase transition, the so-called Berezinsky-Kosterlitz-Thouless (BKT) phase transition. 
At the temperature of a BKT-transition the correlation radius diverges, however the character of a divergence is other than in three-dimensional magnetics.

The apparent inconsistency of the obtained results with classical ones can be eliminated as follows.

It is necessary to remind that the results about the absence of the magnetic order in two-dimensional magnetics are valid for systems with the spontaneously violated continuous symmetry. In our case, considered by us, the presence of a great one-ion anisotropy means that the system has a discrete symmetry group. For systems with the violated discrete symmetry the above mentioned reasonings are not applicable.

Note that the authors [12] have described the BKT-QU phase transition in strongly anisotropic easy-plane ferromagnetic and have found that $T_{\mathrm{c}} \propto\langle S\rangle$, where $\langle S\rangle \ll 1$. Since in our case $\langle S\rangle=0$ we do not consider the vortex phases.

\section{Acknowledgments}

The authors express sincere gratitude to Professor B.A. Ivanov and Dr. C.N. Alexeyev for fruitful discussion and useful remarks.

\section{References}

[1] N. Mermin, H. Wagner, Phys. Rev. Lett. 17, 1133 (1966).

[2] J. Goldstone, Nuovo Cimento 19, 154 (1961).

[3] V.L. Berezinsky, Sov. Phys.-JETP 61, 1144 (1971).

[4] J.M. Kosterlitz, D.J. Thouless, J. Phys. C 6, 1181 (1973).

[5] S.V. Maleev, Sov. Phys.-JETP 70, 2344 (1976).

[6] B.A. Ivanov, E.V. Tartakovskaya, JETP Lett. 63, 792 (1996).

[7] F.P. Onufrieva, Solid State Phys. 23, 2664 (1981).

[8] R.O. Zaytsev, Sov. Phys.-JETP 68, 207 (1975).

[9] Yu.N. Mitsay, Yu.A. Fridman, D.V. Spirin, C.N. Alexeev, SSU Transactions 7, 139 (1998).

[10] Yu.N. Mitsay, Yu.A. Fridman, Theor. Math. Phys. 89, 263 (1989).

[11] V.V. Valkov, T.A. Valkova, preprint N 667F, Krasnoyarsk 1990.

[12] B.A. Ivanov, D.D. Sheka, Fiz. Nizk. Temp. 21, 431 (1995). 